\title{
Prediction of the collapse modes of PVC cylindrical shells under compressive axial loads using Artificial Neural Networks
}

\author{
Angelos P. Markopoulos, Dimitrios E. Manolakos, and Nikolaos M. \\ Vaxevanidis \\ National Technical University of Athens, \\ Manufacturing Technology Division \\ 9 Iroon Polytechniou Avenue, 15780, Athens, Greece \\ E-mail: manolako@central.ntua.gr, \\ WWW home page: http://users.ntua.gr/manolako \\ 2 Department of Mechanical Engineering Teachers, Institute of \\ Pedagogical and Technological Education, N. Heraklion Attikis, Greece, \\ E-mail: vaxev@central.ntua.gr
}

\begin{abstract}
In the present paper Artificial Neural Networks (ANN) are applied in order to predict the buckling modes of thin-walled PVC tubes under compressive axial forces. For the development of the models the neural network toolbox of Matlab ${ }^{\circledR}$ was applied. The results show that these models can satisfactorily face these problems and they constitute not only a fast method, compared to time consuming experiments, but also a reliable tool that can be used for the studying of such parts which are usually employed as structural elements for the absorption of the energy of an impact, in automotive and aerospace applications.
\end{abstract}

\section{Introduction}

Due to technological, managerial and legal implications, nowadays, there is an increased interest in the field of safety and protection of the passengers in the case of a car accident. Therefore, automotive industries are focused even more on the damages that a collision imposes on the vehicle. The simulation and the analysis of a vehicle crash are extremely complicated due to the simultaneous interaction of many elements. The various components are structural elements of plain geometric shape, such as thin-walled tubes, cones etc., designed to absorb the impact energy by deforming plastically, thus protecting the passengers, the vehicle and the load.

For the determination of the energy absorption capacity and the response of these elements a simulative test, i.e. the axial crash of thin-walled components is used; by 
this way the deformation characteristics of the components are identified [1-3]. The combined influence of component's geometry and material on their behavior during the crash is of utmost importance. Moreover, the static or dynamic nature of loads should be taken into account. Most of the cases are studied using static analysis, since it is proven that phenomena occurring under dynamic loads are similar to the ones observed under static loading. The overall behavior of the structure depends on the plastic deformation of whole or part of the structure. Taking into account all these factors, the importance and the necessity for further research in that area, in order to fully understand the phenomena involved, become obvious.

The present paper deals with the modeling of the collapse of PVC cylindrical shells under the effect of compressive axial forces, using ANN models. This type of artificial intelligence is applied to a great number of areas of technology, especially for problems where the input and output values cannot be directly connected by simple equations, as in the case of manufacturing $[4,5]$. Therefore, the topic studied in this paper is quite challenging since the connection between input and output values is rather complicated. Note that to the authors' knowledge, applications of ANN to crashworthy response are very few, if any. As far as the structure of the paper is concerned, first an overview of the plastic deformation of cylindrical shells and a brief description of neural networks is given. Then the experiments performed in order to acquire the data for the training of the neural network are described. Finally, the neural network models and their results are presented and discussed thoroughly.

\section{Plastic deformation of cylindrical shells}

Thin-walled shells collapse plastically under the effect of compressive loads and create folds (buckles) when the stress at a point exceeds the critical yield point. The phenomenon of folding can be analyzed into two phases: the pre-buckling and the post-buckling phase. The post-buckling phase is the one that contributes the most to energy absorption, therefore is the one that will be analyzed the most hereafter.

In the post-buckling phase the shell is plastically deformed, resulting to the creation of buckles and its progressive collapse; the characteristic fluctuation of the axial force is caused by the formation of these buckles. The points of maximum and minimum stress in a stress-strain curve, obtained from the compression of a tube, are respectively the points where the buckling starts and ends. The transitional point at the beginning of the shell's plastic collapse describes the maximum load that the shell can withstand. However, in the dynamic analysis of the collapse of tubes the evaluation of capability of energy absorption of the shell is more important than the value of the maximum load.

There is a variety of modes by which a shell may collapse; the occurrence of a mode depends upon geometric parameters such as the ratios $\mathrm{D} / \mathrm{t}$ (diameter / thickness), L/D (length / diameter) and the properties of the material. In general, the following collapse modes are identified:

(a) Concertina mode: The cylindrical shell, under the effect of the external load, collapses at the shape of concertina with successive axisymmetric folds of length 
equal to $2 \mathrm{~h}$, i.e., buckling wave length; this mode was studied by Alexander [6]. Models for concertina mode have been also proposed by Jones and Abramowicz [7] and Gupta and Velmurugan [8].

(b) Diamond mode: The shell collapses in a non-symmetrical way formatting successive non extensible triangular or trapezoid lobes. The shape of its cross-section can be changed in elliptical, triangular, square and generally polygonal shape resembling the form of a diamond. This buckling mode has been studied extensively by Johnson et al. [9].

(c) Mixed mode: Initially the shell collapses in concertina mode but after the formation of a number of axisymmetric lobes, diamond collapse takes place. The characteristic curve of axial load-axial displacement of mixed mode is a combination of the characteristic curves of concertina and diamond mode; see [1].

(d) Euler buckling: The shell behaves like a bending strut. The work that is absorbed by the shell when Euler buckling occurs is minimal comparing with the work absorbed when other collapse modes take place. Therefore, it is desirable to avoid this particular mode.

(e) Other modes: In this category other collapse modes, such as tilting of the shell's axis, are included. Shearing of one or both ends of the shell that are in contact with the plates may occur, leading to the displacement of one end.

As it has already been mentioned, the mode that a shell develops when it collapses depends upon the material and its geometrical characteristics, namely the thickness (t), the diameter (D) and the length (L). Actually, the collapse mode for a given material depends upon the ratios t/D and L/D [2]. It has been established that shells with small length and large thickness collapse in concertina mode, while shells with large length and small thickness collapse in diamond mode. The transitional area between these two modes has not been clearly determined [1, 2]. Nevertheless, other collapse modes may occur during the compaction of the tubes. The model developed in the present paper is capable of determining the collapse mode when the geometrical and material characteristics of the tube are given.

\section{Experimental results}

Shells of PVC were examined; the geometrical dimensions of the shells, i.e. the ratios $t / D$ and $L / D$, where $t, D$ and $L$ are the thickness, the outer diameter and the length of each tube respectively were considered as variables. The compression was performed between two steel parallel plates, by using an Instron universal testing machine. The plates' velocity was about $10 \mathrm{~mm} / \mathrm{min}$. In order to cover a wide range of values, 24 experiments were carried out ranging between $0.0470 \leq t / D \leq 0.1410$ and $0.92 \leq \mathrm{L} / \mathrm{D} \leq 3.94$. For each experiment the collapse mode was identified and logged.

\section{ANN models}

For the development of the ANN models the neural networks toolbox of Matlab ${ }^{\otimes}$ was used. In this program the creation of neural networks is simplified by using a 
small amount of commands; the program has a data base with functions, algorithms and commands for this purpose. For an insight to the ANN method and Matlab ${ }^{\circledR}$, Refs $[10,11]$ are suggested.

The model presented aims to the prediction of the collapse mode of a tube made from PVC when the t/D and L/D ratios are given. This implies that the two ratios are the input data of the models and the collapse mode the output. Due to the fact that the collapse mode is not an arithmetic value and therefore it cannot be inserted into the program, a substitution becomes necessary; numerals 1, 2, 3 and 4 which stand for Diamond mode, Mixed mode, Concertina mode and Euler buckling, respectively, are introduced.

After the determination of the size of the input and output layers the number of the hidden layers and the neurons within these layers must be decided. This task is accomplished by a trial procedure where different architectures are tested and the one providing the best possible results is finally selected. It is already known that a more complicated than needed network has a reduced generalization capability, since it is characterized by complicated relations. Therefore trials are constrained in models having one or two hidden layers. In the case of one hidden layer models, they are created and tested having $2,3,4,5,6,7,8$ and 9 neurons at the hidden layer. In the case of two hidden layers, the models are created having $2,3,4,5,6,7$ and 8 neurons at the first layer and 3, 4, 5, 6, 7 and 8 at the second hidden layer and all combinations between them are tested; a total of 56 models created. Similarly to the case of selecting the number of hidden layers and their neurons number, for selecting training algorithms trials are made. The training algorithms that are used in the models are the back-propagation algorithm with variable training rate and use of momentum factor and the Levenberg-Marquardt algorithm. Therefore, in total 112 models are created and tested.

As activation function in the hidden layers the hyperbolic tangent sigmoid transfer function was used. It is defined as a strictly increased function, which maintains balance between linear and non-linear behavior and its use provides the opportunity of forming non-linear relations, resulting to the potential of solving nonlinear problems.

For the improvement of generalization of the suggested neural network the early stopping technique was used. In this method the existing data are separated in three subsets. The first subset consists of the training vectors, which are used to calculate the gradient and to form the weight factors and the bias. The second subset is the validation group. The error in that group is observed during training and likewise training group normally decreases during the initial phase of training. However, when the network begins to adjust the data more than needed, the error in that group raises and when that increase is continued for a certain number of repetitions, training stops in order to avoid over-fitting. Finally, the third subset is the test group and its error is not used during training. It is used to compare the different models and algorithms.

The selection of each group's data must be representative and uniform. The training group is the biggest and the test group the smallest among the groups. During the training it is possible to watch the change of the MSE of each group versus the epochs graphically. It is known that the original values given to bias and 
weight factors are chosen randomly each time a network is trained. So training ends up each time in different values of these factors and hence in different error values. For that reason training of each model takes place more than one time; each model is trained five times in order to clearly determine whether a model truly converges to a low value or it is a false value, because e.g. the model was trapped in a local minimum of the function. The mean value of the MSE for these 5 repetitions is calculated and by comparing this mean value the more appropriate one is chosen; that is the model with the lowest mean MSE value in the test group. The error in the test group is more indicative of the generalization capability of the model.

\section{Results and Discussion}

In Figs 1 (a) and (b) the mean MSE of the training and test groups of all the models trained with the back propagation and the Levenberg-Marquardt algorithm are presented. For the recognition of the models a kind of code is adopted. The models are named according to their architecture, by 1 or 2 numbers depending on the number of the hidden layers they have, where each number is the amount of neurons in the corresponding layer. Furthermore, the numbers are accompanied by letters, by $\mathrm{gdx}$ or $\mathrm{lm}$, depending on the training algorithm used for their training, where the first one corresponds to the back-propagation and the second to the Levenberg-Marquardt algorithm. For example the 5 -gdx model is the one with one hidden layer with 5 neurons trained with the back-propagation algorithm and the 6-8-1m model has two hidden layers with 6 and 8 neurons respectively, trained with the LevenbergMarquardt algorithm.

From these charts the model with the minimum test group MSE is selected as the optimal model. It can be seen that this model is the 7- $1 \mathrm{~m}$. In particular, the repetition with the lower test MSE among the 5 values is the one saved and used for the simulation.

In general, the MSE of the models built was relatively small. Models trained with the back-propagation algorithm result to greater errors than those trained with the Levenberg-Marquardt algorithm. Models trained using Levenberg-Marquardt algorithm and have one hidden layer with 5 or more neurons in it, result to errors similar between them with the smallest to be the 7-1m. Nevertheless, models with two hidden layers also provided accepted results, considering that the particular problem which was simulated using neural networks is quite complicated. The creation of a single network for the simulation of two materials with different properties and behavior concerning to crash reaction, is another factor that makes the procedure of finding relations between the variables even harder and more complicated.

The number of epochs needed to complete the training of each model was small, while by comparing the algorithms used, training with Levenberg-Marquardt algorithm required fewer epochs to complete training. This is expected, since it is known that the particular algorithm has the larger convergence speed. Furthermore, a criterion of maximum epochs was used when programming the models and the limit was set to 600 epochs. In all cases, training was not terminated using the criterion of 
maximum number of epochs, since none of the trials needed more than 600 epochs to complete, which is attributed, mainly, to the use of the early stopping technique. More specifically, Levenberg-Marquardt algorithm, in most of the cases completed training after 10-20 epochs, while the maximum number of epochs needed was 71 . Training using the back-propagation algorithm ranged in higher levels and the larger testing occurred after 143 epochs.

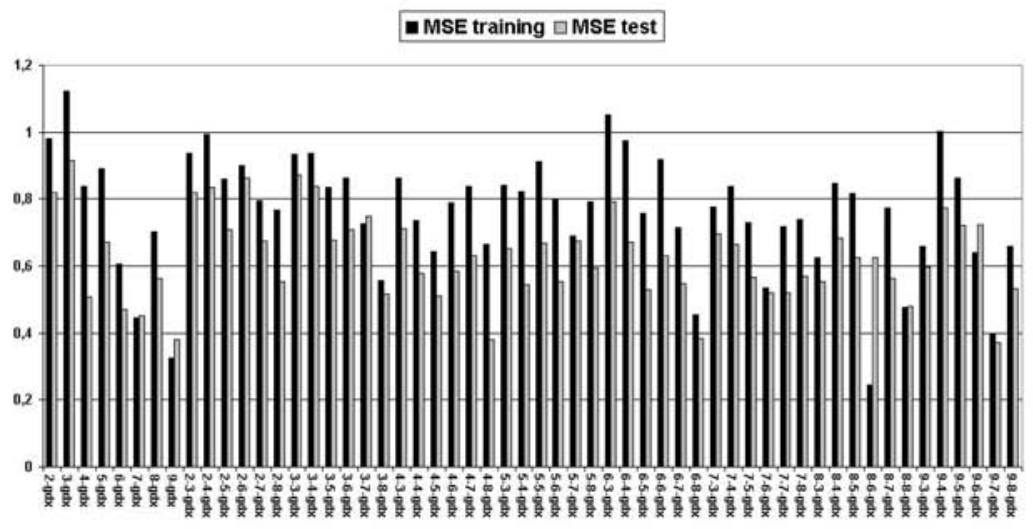

(a)

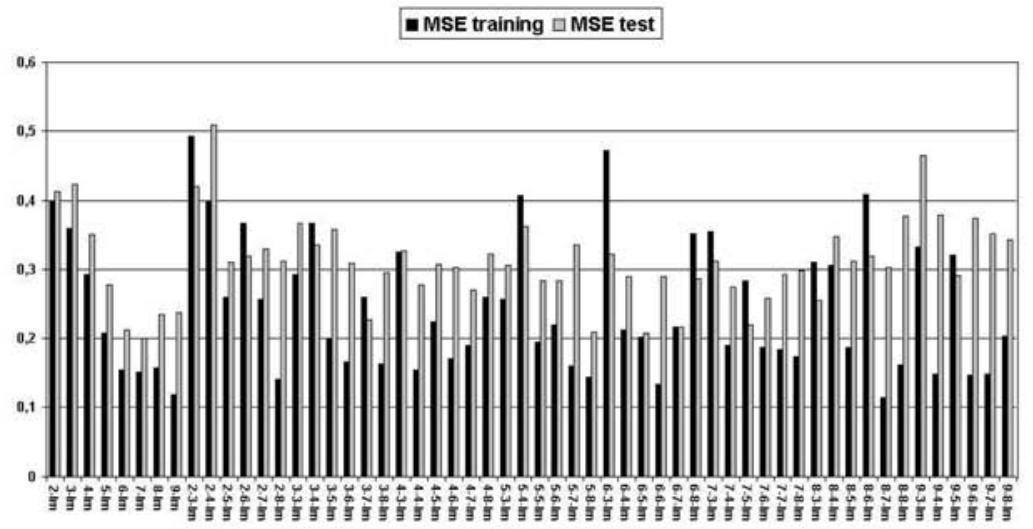

(b)

Fig. 1. Mean value (of the five trainings) of the MSE of training and test groups of models trained using (a) the back-propagation and (b) the Levenberg -Marquardt algorithm.

Note, that the desired output values of the model are integers from 1 to 4 , implying that the numbers resulting from a model are automatically rounded to the nearest integer in order to provide the output. When the program was tested with values that were not inserted in any of the three groups provided during training it predicted correctly the value corresponding to a collapse mode. 
With the results of the trained ANN classification charts for collapse modes for PVC cylindrical shells can be constructed, which indicate the areas of collapse modes and the transitional borders from one mode to another, see Fig 2.

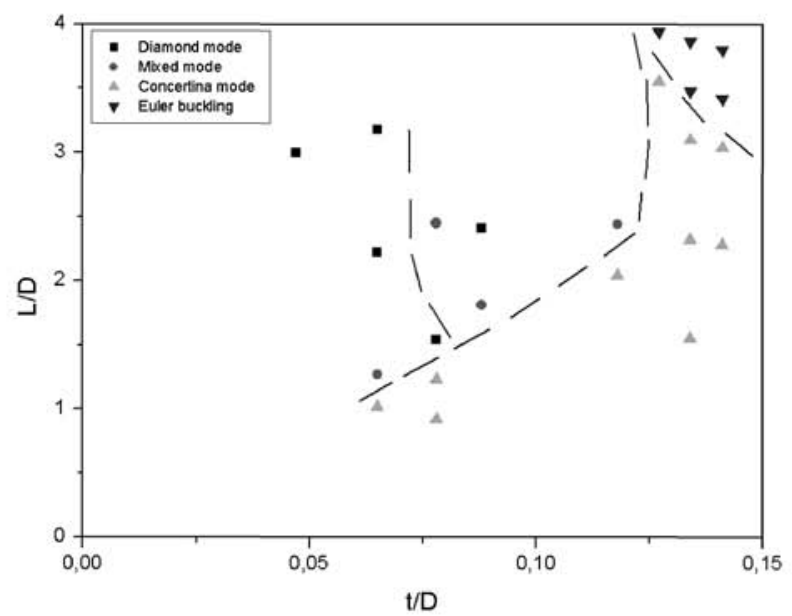

Fig. 2. Classification chart for collapse modes of PVC tubes.

By observing the charts the following conclusions can be drawn: the diamond mode is favored when the $t / D$ ratio is small and the $L / D$ ratio increases. For medium values of the ratios mixed mode is favorable and for even larger ratio values the Euler buckling takes place. In the other regions concertina mode prevails. The classification charts presented here are consistent with others already reported in the relative literature $[1,12]$.

\section{Conclusions}

In the present paper neural network models were proposed which can successfully predict the collapse mode of cylindrical shells subjected to axial compressive forces. When the collapse mode of a particular shell is known the mean load that the shell can withstand may be analytically calculated. These shells are mainly used as energy absorbing devices during a potential impact or crash and are of utmost importance for automotive industries.

Taking into account the results of the neural networks models, many points mentioned in the theory were confirmed. Particularly, as it was expected, models trained using the Levenberg-Marquardt algorithm resulted into smaller error in shorter time than the ones trained with the back-propagation algorithm.

The development of neural networks using Matlab ${ }^{B}$ is relatively simple using a small amount of commands. Furthermore, training of the models using the early stopping technique has a very small duration; the maximum is a few seconds, for all 
training algorithms. Training becomes even faster when the Levenberg-Marquardt algorithm is used.

The network's results are considered very good for the particular application since the network is quite complicated. In many cases the collapse mode varies, even only with a minimal alteration of one of the input variables. Moreover, sometimes there is not a particular sequence in the way that collapse modes change and that makes the simulation of the network even harder. However, according to the results obtained, the proposed ANN may be considered a reliable tool for predicting collapse modes. It is expected that models such as the one suggested here can reduce the cost of product development by reducing time and amount of experiments needed.

\section{References}

1. A.G. Mamalis and D.E. Manolakos, Deformation Characteristics of Crashworthy Components (VDI, 1989).

2. K.R.F. Andrews, G.L. England and E. Ghani, Classification of the axial collapse of cylindrical tubes under quasi-static loading, International Journal of Mechanical Sciences, Vol. 25, No. 9-10, 687-696 (1983).

3. S.R. Guillow, G. Lu and R.H. Grzebieta, Quasi-static axial compression of thin-walled circular aluminium tubes, International Journal of Mechanical Sciences 43, 2103-2123 (2001).

4. G. Dini, Literature database on applications of artificial intelligence methods in manufacturing engineering, Annals of the CIRP, 46 (2), 681-690 (1997).

5. A. Markopoulos, N.M. Vaxevanidis, G. Petropoulos and D.E. Manolakos, Artificial Neural Networks Modeling of Surface Finish in Electro-Discharge Machining of Tool Steels, Proc. of ESDA 2006, $8^{\text {th }}$ Biennial ASME Conference on Engineering Systems Design and Analysis, Torino, Italy (July 4-7, 2006).

6. J.M. Alexander, An Approximate analysis of the collapse of thin cylindrical shells under axial loading, Quart. Journal of Mech. And Applied Math., Vol. XIII, Pt. 1 (1960).

7. N. Jones and W. Abramowicz, Static and dynamic axial crushing of circular and square tubes, In: S.R. Reid "Metal forming and Impact Mechanics", Oxford, Pergamon Press, p. 225 (1985).

8. N.K. Gupta and R. Velmurugan, Consideration of internal folding and non-symmetric fold formation axi-symmetric axial collapse round tubes, Int. J. Solid Structures, Vol. 34, 2611-2630 (1997).

9. W. Johnson, P.D. Soden and S.T.S. Al-Hassani, Inextensional Collapse of thin-walled tubes under axial compression, J. Strain Analysis, Vol. 12, No. 4 (1977).

10. S. Haykin, Neural networks, a comprehensive foundation (Prentice Hall, 1999).

11. H. Demuth and M. Beale, Neural networks toolbox for use with Matlab, (User's guide 2001).

12. A.A.A. Alghamdi, Collapsible impact energy absorbers: an overview, Thin-Walled Structures, Vol. 34, No. 2, 189-213 (2001). 